\title{
The Charlson Comorbidity Index Predicts Survival after Disease Recurrence in Patients following Radical Cystectomy for Urothelial Carcinoma of the Bladder
}

\author{
Roman Mayr ${ }^{a}$ b Matthias May ${ }^{c}$ Maximilian Burger ${ }^{a}$ Thomas Martini ${ }^{b, d}$ \\ Armin Pycha $^{b}$ Christopher Dechet ${ }^{b, e}$ Michele Lodde $^{b}$ Evi Comploj ${ }^{b}$ \\ Wolf F. Wieland ${ }^{\mathrm{a}}$ Stefan Denzinger $^{\mathrm{a}}$ Wolfgang Otto $^{\mathrm{a}}$ Atiqullah Aziz $^{\mathrm{a}}$ \\ Hans-Martin Fritsche ${ }^{a}$ Michael Gierth ${ }^{a}$ \\ ${ }^{a}$ Department of Urology, Caritas St. Josef Medical Center, University of Regensburg, Regensburg, Germany; \\ ${ }^{b}$ Department of Urology, Central Hospital of Bolzano, Bolzano, Italy; ' Department of Urology, St. Elisabeth Hospital, \\ Straubing, ${ }^{\mathrm{d}}$ Department of Urology, University of Mannheim, Mannheim, Germany; ${ }^{\mathrm{e}}$ Huntsman Cancer Institute, \\ Division of Urology, University of Utah, Salt Lake City, Utah, USA
}

\section{Key Words}

Bladder cancer · Distant recurrence $\cdot$ Local recurrence .

Time to recurrence - Outcome - Transitional cell carcinoma . Comorbidity scores

\footnotetext{
Abstract

Objective: To identify prognostic clinical and histopathological parameters, including comorbidity indices at the time of radical cystectomy ( $\mathrm{RC})$, for overall survival (OS) after recurrence following $\mathrm{RC}$ for urothelial carcinoma of the bladder (UCB). Materials and Methods: A retrospective multicenter study was carried out in 555 unselected consecutive patients who underwent RC with pelvic lymph node dissection for UCB from 2000 to 2010. A total of 227 patients with recurrence comprised our study group. Cox proportional hazards regression models were calculated with established variables to assess their independent influence on OS after re-
}

\section{KARGER}

E-Mail karger@karger.com

www.karger.com/uin currence. Results: The median time from $\mathrm{RC}$ to recurrence and the median OS after recurrence was 10.9 and 5.4 months, respectively. Neither the time to recurrence nor the type of recurrence (systematic vs. local) was predictive of the OS. In contrast, age (hazard ratio $(H R) 1.53, p=0.011$ ), lymph node metastasis (HR 1.56, $\mathrm{p}=0.007$ ), and positive surgical margins (HR 1.53, $p=0.046$ ) significantly affected the OS after disease recurrence. In addition, the dichotomized Charlson comorbidity index ( $\mathrm{CCl}$; dichotomized into $>2$ vs. $0-2$ ) was the only comorbidity score with an independent prediction of OS (HR $1.41, p=0.033)$. We observed a significant gain in the base model's predictive accuracy, i.e. from 68.4 to $70.3 \%$ ( $p<$ 0.001), after inclusion of the dichotomized CCI. Conclusions: We present the first outcome study of comorbidity indices used as predictors of OS after disease recurrence in patients undergoing RC for UCB. The $\mathrm{CCl}$ at the time of RC had no significant influence on the time to recurrence but represented an independent predictor of OS after disease recurrence.

(c) 2014 S. Karger AG, Base 


\section{Introduction}

Radical cystectomy (RC) with pelvic lymph node dissection constitutes the gold standard treatment for muscleinvasive urothelial carcinoma of the bladder (UCB) and high-risk non-muscle-invasive disease refractory to instillation therapy $[1,2]$. Despite this aggressive treatment, subsequent disease recurrence occurs in approximately half of patients with muscle-invasive bladder cancer in the first 24 months after RC $[1,3]$. Over two thirds of patients with disease recurrence die within 1 year of recurrence $[4$, 5]. Various clinicopathological parameters have been identified as predictors of overall survival (OS) in patients who experience disease recurrence after RC [4]. In particular, systemic inflammatory parameters, symptoms at recurrence, and a shorter time to recurrence have been described to be independent predictors of OS after recurrence [4-7].

Comorbidity is an important independent prognostic factor for outcome prediction in patients with several oncological diseases [8]. Evidence exists that comorbidity indices predict the cancer-independent survival and the 90-day mortality after RC $[9,10]$. The Eastern Cooperative Oncology Group (ECOG) performance status has been described as a predictor of the OS after disease recurrence following RC [7].

The aim of our multicenter study was to analyze the performance of the most commonly used comorbidity indices [Adult Comorbidity Evaluation-27 (ACE-27), Charlson comorbidity index (CCI), ECOG performance status, and American Society of Anesthesiologists (ASA) score] defined at the time of $\mathrm{RC}$ in order to predict the oncological outcome in UCB patients who experience disease recurrence after RC.

\section{Patients and Methods}

\section{Study Population}

This was an institutional review board-approved study. This retrospective study included 555 unselected consecutive patients who underwent RC with pelvic lymph node dissection for muscleinvasive or recurrent high-risk non-muscle-invasive UCB without distant metastases at 2 tertiary urological centers between 2000 and 2010. In total, 227 patients experienced disease recurrence confirmed by imaging studies (CT, MRI, and/or bone scan) and/or histological verification. RC and bilateral pelvic lymph node dissection were performed by several surgeons. No patient underwent neoadjuvant chemotherapy and/or radiotherapy.

Comorbidity and Performance Indices

Five indices were assessed at the time of RC: ASA, ACE-27, CCI, age-adjusted CCI (ACCI), and ECOG as described previously $[9,10]$. The ASA score was obtained prospectively by anesthesi- ologists in both institutions. According to the current literature, all indices were dichotomized for better clinical applicability in the following manner: ASA, 1-2 versus 3-4; ECOG, 0-1 versus 2-3; CCI, $0-2$ versus $>2$; ACCI, $0-5$ versus $>5$, and ACE-27, $0-1$ versus $2-3$ as well as $0-2$ versus 3 . The CCI, ACCI, and ACE- 27 were calculated without taking UCB into account [8-10].

\section{Outcome Measures}

The study goals were to examine the OS after disease recurrence and to determine the individual predictive accuracy (PA) gain in the aforementioned comorbidity scores in multivariable outcome models. Early recurrence was defined as tumor recurrence within 12 months after RC. The type of recurrence was classified as local or distant disease $[5,6]$. The time of death was verified by local cancer registries and by the treating physician. The histological parameters used for univariable and multivariable analyses were soft tissue surgical margin (STSM), lymphovascular invasion (LVI), and concomitant carcinoma in situ (CIS). All surgical specimens were processed according to standard pathological procedures and evaluated by experienced genitourinary pathologists at each institution. The tumor stage was assigned according to the 7th edition of the TNM classification (2009) [11]. The histopathological grade was based on the 1973 WHO classification. Since all of the study patients had undifferentiated grading, this criterion was not considered in the analysis. A positive surgical margin was defined when tumor was present in the inked areas of the RC specimen, with the exception of tumor presence at urethral and ureteral margins. LVI was defined as tumor cells in an endothelium-lined space without discrimination between L1 and V1. If any disagreement regarding pathological specimens was observed, the original histopathology report was rereviewed by independent pathologists. For better clinical practicability, age was dichotomized with a cutoff at 75 years, which is the most applied cutoff age in patients undergoing RC $[12,13]$. Follow-up regimens after RC were conducted according to institutional protocols and following current guidelines. Patients were seen every 3-4 months for the first year after RC, every 6 months during years $2-5$, and annually thereafter. Diagnostic imaging of the abdomen and/or upper tracts and chest radiography were performed at least annually or when clinically indicated.

\section{Statistical Analyses}

Continuous metric variables are reported as medians with interqurtile range (IQR). Pertinent characteristics of early and late recurrence were compared. The Wilcoxon-Mann-Whitney test was used for nonnormally distributed continuous variables and comparisons between categorical variables were made using Fisher's exact test and a $\chi^{2}$ test.

OS rates after recurrence were estimated using the KaplanMeier method. The log-rank test was employed to compare survival curves. Univariable and multivariable Cox proportional hazards regression analyses were performed to assess the influence of several clinical parameters (including 5 comorbidity and performance indices, age, and BMI) and pathological parameters. For this purpose, a multivariable Cox regression model was created using the stepwise backward elimination process. To assess the PA of the various comorbidity indices, the inclusion of a comorbidity index in a base model (without integration of comorbidity scores) was used. Assessment of the AUC values (c-indices) for estimation of the PA according to Harrell et al. [14] was performed. 
Table 1. Demographic data classified by time of recurrence ( $\leq 12$ vs. $>12$ months) after RC in 227 recurrent patients with UCB

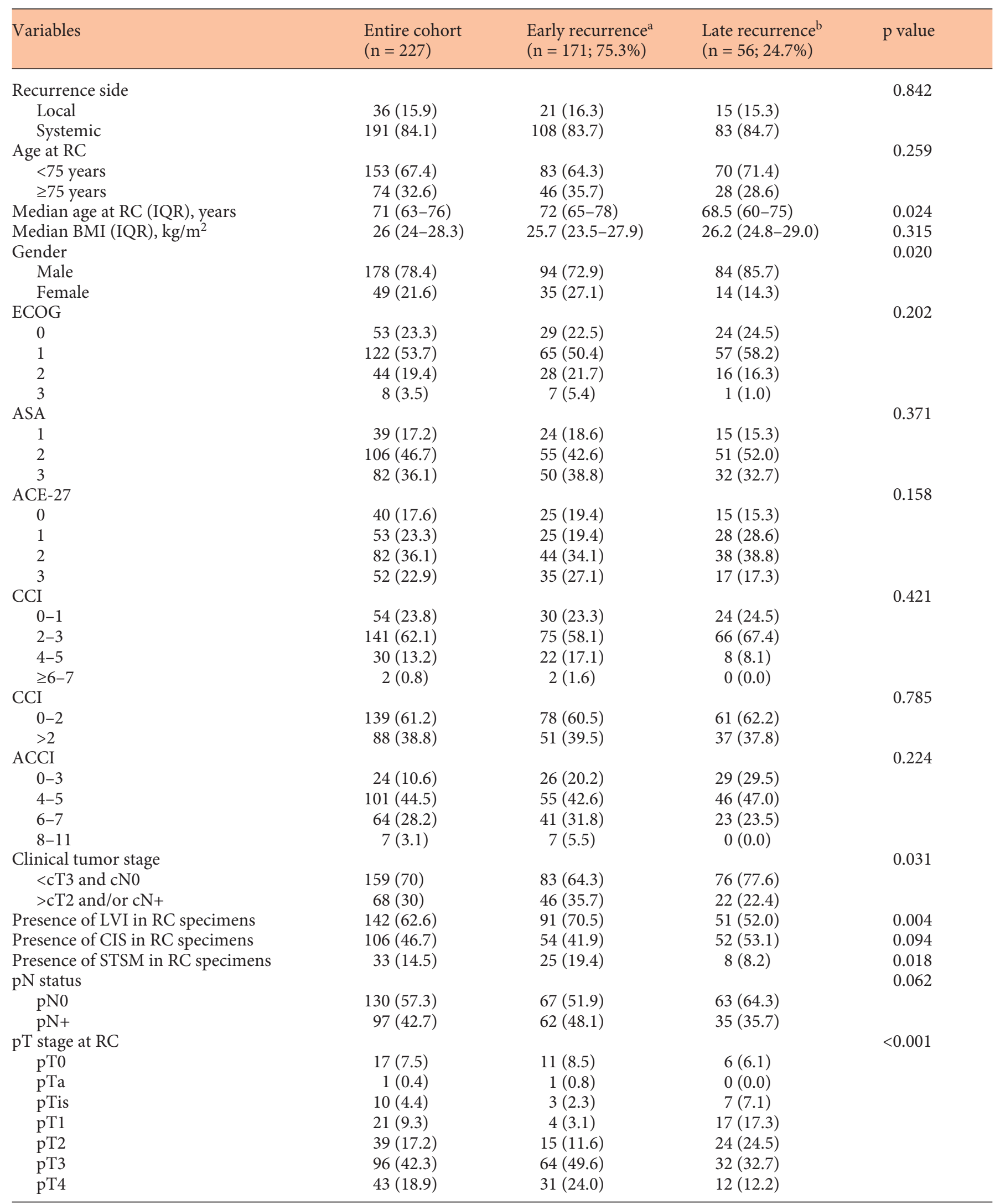


Table 1. (continued)

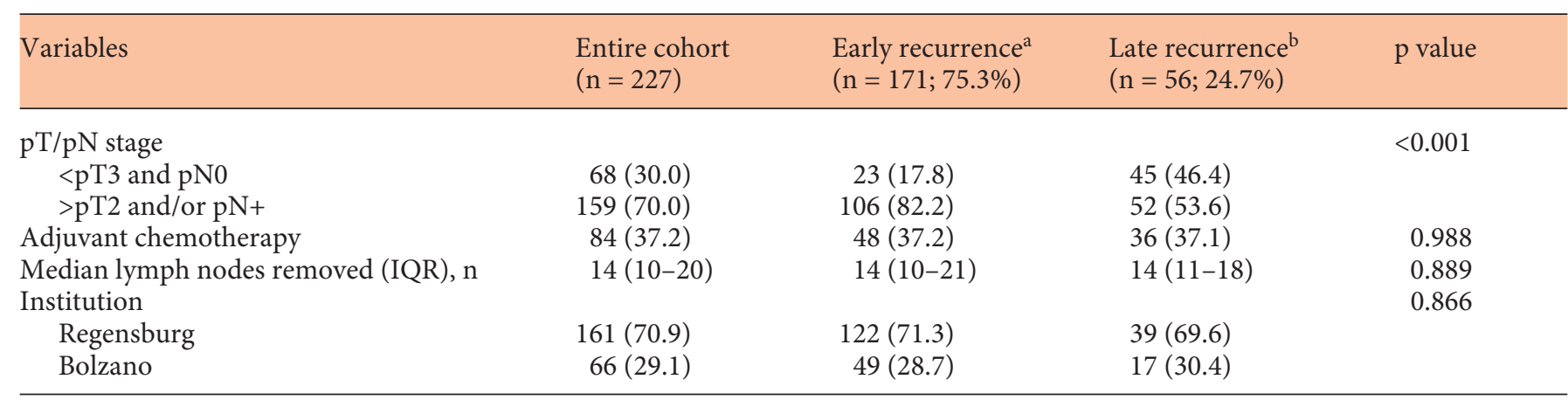

Values are presented as numbers (\%) unless otherwise stated. ${ }^{\mathrm{a}} \leq 12$ months. ${ }^{\mathrm{b}}>12$ months.

The c-indices of the base model with and without the respective comorbidity index were compared according to the MantelHaenszel test [15].

The reported $\mathrm{p}$ values are 2 -sided, and $\mathrm{p}<0.05$ was considered statistically significant. Data were analyzed using SPSS 19.0 (IBM Corp., N.Y., USA).

\section{Results}

\section{Study Population}

The demographic characteristics of 227 study patients with recurrence after $\mathrm{RC}$ and their distribution in relation to the time point of recurrence are shown in table 1 . The median time from RC to recurrence was 10.9 months (IQR $5-24)$. The median age at the time of $\mathrm{RC}$ was 71.0 years (IQR 63-76). Patients with early recurrence ( $\leq 12$ months) after RC were older at RC $(\mathrm{p}=0.024)$, had a higher clinical tumor stage $(\mathrm{p}=0.031)$, had higher rates of LVI $(\mathrm{p}=0.004)$, and had higher positive margin rates $(\mathrm{p}=0.018)$ in their RC specimens. Females made up a higher proportional percentage of the patients suffering an early versus a late recurrence ( 27 vs. $14 \%$, respectively; $\mathrm{p}=0.020$ ). There was no varied distribution between the early- and late-recurrence subgroups for all assessed comorbidity indices. In particular, the dichotomized CCI was not associated with early or late recurrence of UCB after RC $(p=0.785)$. On the other hand, patients with a CCI of $0-2$ had significantly lower systemic recurrence rates compared to patients with CCI $>2$ (79.1 and 92.0\%, respectively; $\mathrm{p}=0.009$ ).

\section{Univariable Analyses Predicting OS after Recurrence}

The median OS after recurrence was 5.4 months. After $6,12,36$, and 60 months, 37.4, 21.1, 5.7, and $0.4 \%$ of the entire study group were alive, respectively. Neither the time to recurrence nor the location of recurrence was a significant predictor of OS after disease recurrence ( $\mathrm{p}=$ 0.806 and $\mathrm{p}=0.108$, respectively; fig. $1 \mathrm{a}, \mathrm{b}$ ). The dichotomized CCI ( $>2$ vs. $0-2$ ) was a significant predictor of OS in the univariable analysis (HR 1.53, 95\% CI 1.13-2.07, $\mathrm{p}=0.006$; table 2). In particular, patients with a CCI of $0-2$ showed an OS after $6,12,36$, and 60 months of 44.6, $26.6,7.2$, and $0.7 \%$, respectively. In contrast, patients with a CCI $>2$ showed an OS of $26.1,12.5,3.4$, and $1.1 \%$, respectively ( $\mathrm{p}=0.003$; fig. $1 \mathrm{c})$. Details of the univariable analyses of OS after recurrence are listed in table 2 . In particular, neither the kind of recurrence (systemic vs. local) nor the time of recurrence (cutoff 12 months) showed significant differences in survival. Along with the aforementioned dichotomized CCI results, the dichotomized ACCI ( $0-5$ vs. $>5, p=0.002)$, continuous ACCI ( $\mathrm{p}<$ $0.001)$, and ACE-27 (0-2 vs. $>2, p=0.036)$ significantly predicted the OS after disease recurrence (table 2).

\section{Multivariable Analyses Predicting OS after Recurrence}

The results of the multivariable Cox models are shown in table 3. According to a stepwise backward elimination process, independent predictors of OS after recurrence were positive surgical margins (HR 1.53, $\mathrm{p}=0.046$ ), patients older than 75 years (HR 1.53, p = 0.011), lymph node metastasis at RC (HR 1.56, p = 0.007), and CCI $>2$ (HR 1.41, $\mathrm{p}=0.033$ ). The multivariable model containing clinical and histopathological variables with and without the dichotomized CCI showed a PA of 70.3 and $68.4 \%$, respectively. The PA gain was $1.9 \%(\mathrm{p}<0.001)$. Based on its predictive strength and the significant gain in $\mathrm{PA}$, only the dichotomized CCI was included in the Cox model. Other indices were not included as their contribution was not significant (data not shown). 

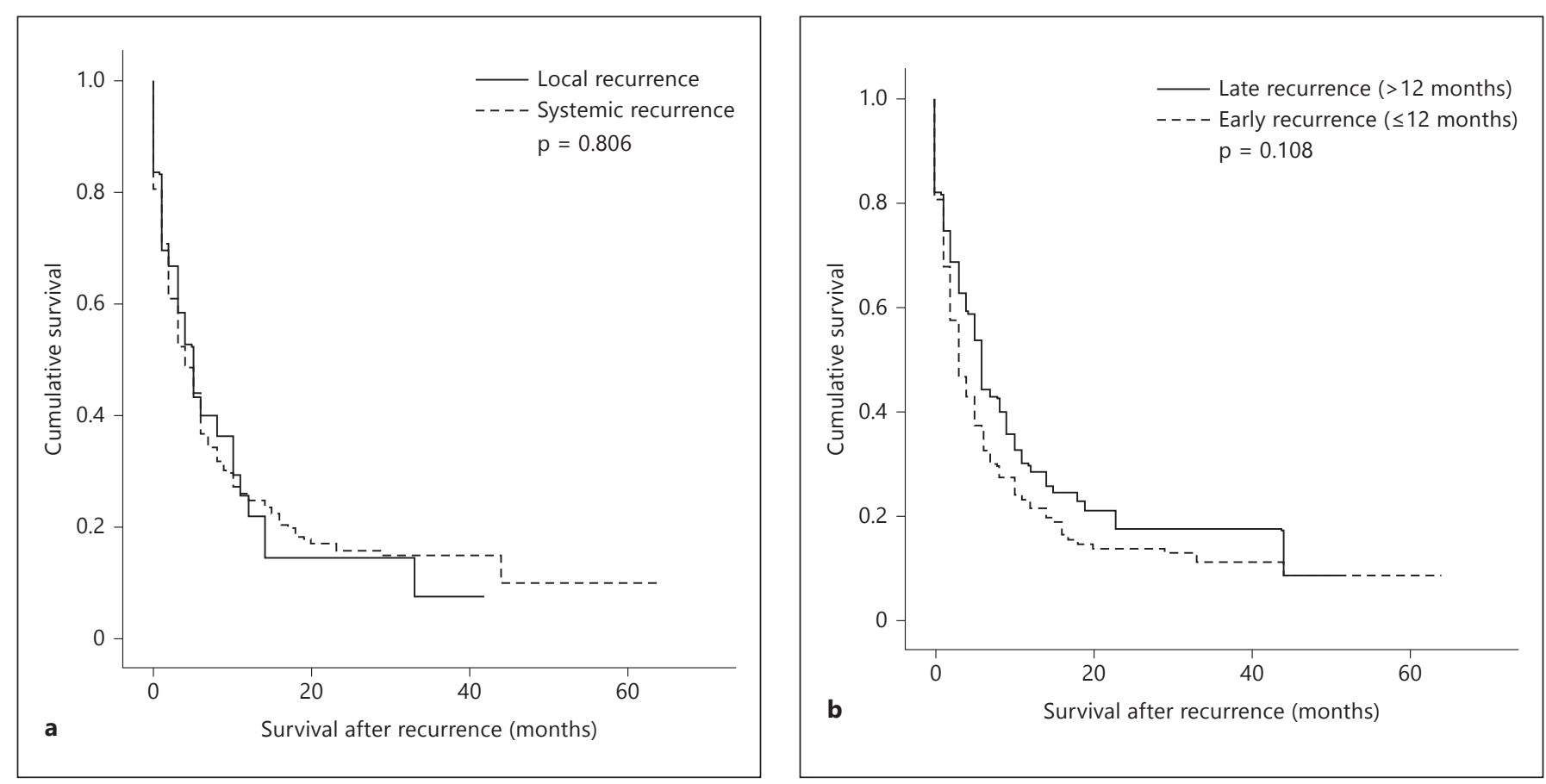

Fig. 1. Kaplan-Meier curves to assess the influence of the kind of recurrence (local vs. systemic (a), the time to recurrence ( $>12$ vs. $\leq 12$ months) (b), and the dichotomized CCI (0-2 vs. $>2)$ (c) on OS after recurrence in patients who underwent RC for UCB.

\section{Discussion}

In this study, 227 patients who experienced recurrence after RC had comorbidity assessment performed at the time of RC using 4 validated comorbidity/performance indices: ASA, CCI, ECOG, and ACE-27. Systemic recur-

rence occurred in $84 \%$ of the study patients, and 3 of 4 study patients developed recurrence within 1 year after RC. Only $6 \%$ of the patients were alive 36 months after disease recurrence, emphasizing the aggressive nature of recurrent UCB. Three main findings emerged from this study. Firstly, the CCI was the only comorbidity index 
Table 2. Univariable Cox regression analyses to assess the association of clinicopathological variables and OS after recurrence in patients who underwent RC for UCB

\begin{tabular}{|c|c|c|c|}
\hline Variables & HR & $95 \% \mathrm{CI}$ & $\mathrm{p}$ value \\
\hline Age (cont.) & 1.031 & $1.014-1.048$ & $<0.001$ \\
\hline Age $\geq 75$ years (ref. $<75$ years) & 1.529 & $1.128-2.074$ & 0.006 \\
\hline Female sex (ref. male) & 1.104 & $0.778-1.556$ & 0.579 \\
\hline BMI (cont.) & 0.996 & $0.960-1.033$ & 0.828 \\
\hline ECOG (cont.) & 1.173 & $0.971-1.415$ & 0.098 \\
\hline ECOG 2-3 (ref. 0-1) & 1.081 & $0.769-1.521$ & 0.652 \\
\hline ASA (cont.) & 1.233 & $0.995-1.526$ & 0.055 \\
\hline ASA 3-4 (ref. 1-2) & 1.288 & $0.953-1.741$ & 0.099 \\
\hline ACE-27 (cont.) & 1.160 & $0.997-1.350$ & 0.055 \\
\hline ACE-27 2-3 (ref. 0-1) & 1.243 & $0.920-1.680$ & 0.157 \\
\hline ACE-27 3 (ref. 0-2) & 1.443 & $1.024-2.033$ & 0.036 \\
\hline CCI (cont.) & 1.103 & $0.976-1.248$ & 0.116 \\
\hline CCI > 2 (ref. 0-2) & 1.528 & -2.065 & 0.006 \\
\hline ACCI (cont.) & 1.183 & $1.076-1.299$ & $<0.001$ \\
\hline ACCI >5 (ref. 0-5) & 1.636 & $1.199-2.232$ & 0.002 \\
\hline pN+ stage (ref. pN0) & 1.578 & $1.167-2.133$ & 0.003 \\
\hline \multicolumn{4}{|l|}{ Number of lymph nodes } \\
\hline & 0.982 & $0.965-0.999$ & 0.041 \\
\hline pT3/4 stage (ref. $\leq$ pT2) & 1.533 & $1.119-2.099$ & 0.008 \\
\hline \multicolumn{4}{|l|}{$>$ pT2 and/or $\mathrm{pN}+$} \\
\hline & 1.902 & $1.337-2.705$ & $<0.001$ \\
\hline LVI present (ref. absent) & 1.684 & $1.218-2.328$ & 0.002 \\
\hline CIS present (ref. absent) & 0.857 & $0.637-1.152$ & 0.306 \\
\hline Positive STSM (ref. negative) & 1.838 & $1.226-2.754$ & 0.003 \\
\hline \multicolumn{4}{|l|}{ Adjuvant chemotherapy } \\
\hline & 0.967 & & 0.825 \\
\hline Systemic recurrence (ref. local) & 0.955 & $0.644-1.415$ & 0.818 \\
\hline \multicolumn{4}{|l|}{ Recurrence $\leq 12$ months (ref. } \\
\hline & 1.325 & $0.918-1.913$ & 0.132 \\
\hline Bolzano (ref. Regensburg) & 1.043 & $0.764-1.425$ & 0.791 \\
\hline
\end{tabular}

cont. = Continuous; ref. = reference; $\mathrm{HR}=$ hazard ratio; $95 \%$ $\mathrm{CI}=95 \%$ confidence interval.

that could increase the PA in a multivariable analysis of OS after disease recurrence. Secondly, the CCI at the time of RC had no impact on the time to recurrence but influenced the type of recurrence, with more systemic recurrences occurring in patients with higher comorbidity. Thirdly, neither the time to recurrence nor the type of recurrence was a significant predictor of the OS after disease recurrence.

The assessment of comorbidity in predicting survival has become increasingly important in oncology [8]. We therefore assessed 4 different indices to evaluate comorbidity at the time of $\mathrm{RC}$ as a predictor of OS after disease recurrence. In the univariable Cox analyses, the continuous and dichotomized ACCI $(\mathrm{p}<0.001$ and $\mathrm{p}=0.002$,
Table 3. Multivariable Cox proportional hazards regression analyses of variables associated with OS after recurrence in patients who underwent RC for UCB

\begin{tabular}{|c|c|c|}
\hline \multirow[t]{2}{*}{ Variables } & \multicolumn{2}{|l|}{$\begin{array}{l}\text { Stepwise backward } \\
\text { elimination }\end{array}$} \\
\hline & HR (95\% CI) & $\mathrm{p}$ value \\
\hline Female sex (ref. male) & - & - \\
\hline Bolzano (ref. Regensburg) & - & - \\
\hline BMI (cont.) & - & - \\
\hline Concomitant CIS (ref. absent) & - & - \\
\hline Positive STSM (ref. negative) & $1.529(1.007-2.321)$ & 0.046 \\
\hline \multicolumn{3}{|l|}{ Number of lymph nodes } \\
\hline removed (cont.) & $0.985(0.967-1.003)$ & 0.103 \\
\hline Adjuvant chemotherapy (ref. no) & - & - \\
\hline Age $\geq 75$ years $($ ref. $<75$ years) & $1.526(1.103-2.112)$ & 0.011 \\
\hline pT3/4 stage (ref. $\leq$ pT2) & - & - \\
\hline $\mathrm{pN}+$ stage (ref. pN0) & $1.562(1.132-2.156)$ & 0.007 \\
\hline LVI present (ref. absent) & - & - \\
\hline Systemic recurrence (ref. local) & - & - \\
\hline $\mathrm{CCI}>2$ (ref. CCI $0-2$ ) & $1.405(1.027-1.921)$ & 0.033 \\
\hline \multicolumn{3}{|l|}{ Recurrence $\leq 12$ months } \\
\hline (ref. $>12$ months) & - & - \\
\hline $\mathrm{AUC}^{\mathrm{a}}$ (with CCI), \% (IQR) & $70.3(61.9-78.7)$ & \\
\hline $\begin{array}{l}\text { Gain in predictive accuracy (ref. } \\
\text { basic model without CCI), \% }\end{array}$ & 1.9 & $<0.001$ \\
\hline
\end{tabular}

cont. = Continuous; ref. $=$ reference; AUC $=$ area under the curve; $\mathrm{HR}=$ hazard ratio; $95 \% \mathrm{CI}=95 \%$ confidence interval. ${ }^{a}$ Without CCI: $68.4 \%$.

respectively), the dichotomized CCI $(\mathrm{p}=0.006)$, and the dichotomized ACE-27 (0-2 vs. 3, p = 0.036) were significant predictors of OS after recurrence. In our multivariable analysis, only the dichotomized CCI could increase the PA of our Cox model, with a PA gain of $1.9 \%$ $(\mathrm{p}<0.001)$. To our knowledge, this is the first study to show that comorbidity is predictive of OS in this patient cohort.

In our study, no significant differences using comorbidity indices were observed in patients experiencing early or late recurrence of UCB (table 1). In particular, patients with early and late recurrences showed no significant differences in the distribution of the dichotomized CCI (CCI 0-2; 60.5 and 62.2\%, respectively; $\mathrm{p}=0.785$ ). Our outcome results are in contrast to prior studies investigating patients after recurrence of UCB. In a recent study by Rink et al. [4], a shorter time to recurrence following RC was identified as a risk factor for poor OS after recurrence, which could not be demonstrated in our cohort. Mitra et al. [6] showed early recurrence ( $<12$ months) 
after RC as an independent predictor of OS after recurrence. Additionally, they assessed the location of recurrence and found distant metastasis and the combination of distant metastasis and local recurrence to be independent risk factors for a shorter OS after recurrence [6]. Furthermore, in a recent report of 114 Japanese patients who experienced recurrence after $\mathrm{RC}$, the detection of recurrence after 1 year was a positive independent predictor of OS after recurrence (HR 0.58, p = 0.021) [5]. Differences in patient inclusion criteria, patient characteristics, follow-up schemes, and patterns of recurrences may have accounted for this discrepancy. Nevertheless, our data confirms that the median time from $\mathrm{RC}$ to disease recurrence is usually $<12$ months (the median time to recurrence was 10.9 months in our study).

A further important finding of the present study is that positive lymph nodes, an age greater than 74 years, and a positive STSM at RC are independent predictors of OS after recurrence (table 3 ). This is in agreement with the current literature $[4,6]$. The results of the above mentioned Japanese single-center study point out that an age over 60 years has a significant detrimental influence on survival after disease recurrence [5]. Positive lymph node status was not faced as a separate variable against lymph node negative status in the study by Mitra et al. [6], but lymph node density $>10 \%$ was significantly associated with a worse OS after recurrence $(\mathrm{p}=0.001)$.

Retrospective studies suggest that the ASA score is a predictor of 90-day mortality, and the CCI and ACE-27 are predictors of cancer-independent mortality in patients after RC of UCB $[9,10]$. In a retrospective study of Novotny et al. [13] consisting of $70 \mathrm{RC}$ patients with an ASA score of 3 and an increased age ( $\geq 75)$, a regular recurrence-free survival but a decreased cancer-independent mortality were observed. In the present study assessing 4 different comorbidity indices, only the CCI was predictive of the OS after disease recurrence. Study patients with CCI $>2$ had, after 6 and 12 months, cancer-specific mortality at higher rates of 18.5 and $14.1 \%$, respectively, in contrast to patients with a CCI of $0-2$. In addition, the integration of the dichotomized CCI in our multivariable model was able to increase the PA significantly.

The present retrospective study had certain limitations that must be considered when interpreting its findings. Firstly, the assessment of the comorbidity scores at the time of RC must be respected. The median time between $\mathrm{RC}$ and recurrence was relatively short at 10.9 months; therefore, a significant change in comorbidity values at the time of recurrence would not be expected. Secondly,

Impact of Comorbidity on Survival after Recurrence our analysis also could not be adjusted for the influence of the kind of therapy after recurrence. It is possible that healthier patients received more aggressive salvage therapy. Thirdly, no information regarding the number of distant or local recurrences or the symptoms at the time of recurrence was available. Nakagawa et al. [5] demonstrated that the number of metastatic organs at recurrence was an independent predictor of OS after RC (HR $2.16, \mathrm{p}=0.006$ ).

Our data suggest that more ill patients $(\mathrm{CCI}>2)$ show a significantly shorter OS after recurrence than their healthier counterparts $(\mathrm{CCI} \leq 2)$. An explanation for this reduced OS after recurrence could be that these patients do not get the same aggressive therapy as healthier patients. We therefore feel that these patients with recurrence after RC should be discussed in an interdisciplinary tumor board review in the same way as their healthier counterparts to offer them the best possible care considering their renal function, performance status, and worse outcomes after recurrence. In addition, the reduced survival of patients with a CCI $>2$ could be explained by a different tumor microenvironment, an impaired immune response, and/or a different metastatic cluster. Further research could be helpful to understand the different tumor microenvironment, tumor immunology, and metastatic cluster in individuals with several comorbidities in order to translate this knowledge into therapeutic strategies for the patients.

In conclusion, we present the first study that shows that comorbidity is an important predictor of OS after disease recurrence in patients undergoing RC for UCB. The dichotomized CCI independently predicted OS, along with age, lymph node metastasis, and positive surgical margins. Specifically, patients with higher CCI scores are at a higher risk of dying from their recurrent disease. We recognize that more studies are necessary to confirm our findings and help to evaluate the full impact of comorbidity on survival. Finding more predictive survival measures such as comorbidity may help to assess patients at a higher risk for poorer survival and to identify candidates for additional salvage therapeutic strategies and clinical trials.

\section{Acknowledgement}

We thank Jenny Wagner for her excellent assistance. 


\section{References}

1 Stein JP, Lieskovsky G, Cote R, et al: Radical cystectomy in the treatment of invasive bladder cancer: long-term results in 1,054 patients. J Clin Oncol 2001;19:666-675.

$\checkmark 2$ Madersbacher S, Hochreiter W, Burkhard F, et al: Radical cystectomy for bladder cancer today - a homogeneous series without neoadjuvant therapy. J Clin Oncol 2003;21: 690-696.

-3 Shariat SF, Karakiewicz PI, Palapattu GS, et al: Outcomes of radical cystectomy for transitional cell carcinoma of the bladder: a contemporary series from the Bladder Cancer Research Consortium. J Urol 2006;176:2414-2422.

$>4$ Rink M, Lee DJ, Kent M, et al: Predictors of cancer-specific mortality after disease recurrence following radical cystectomy. BJU Int 2013;111:E30-E36.

$\checkmark 5$ Nakagawa T, Hara T, Kawahara T, et al: Prognostic risk stratification of patients with urothelial carcinoma of the bladder with recurrence after radical cystectomy. J Urol 2013; 189:1275-1281.
6 Mitra AP, Quinn DI, Dorff TB, et al: Factors influencing post-recurrence survival in bladder cancer following radical cystectomy. BJU Int 2012;109:846-854.

7 Boorjian SA, Tollefson MK, Cheville JC, Costello BA, Thapa P, Frank I: Detection of asymptomatic recurrence during routine oncological followup after radical cystectomy is associated with improved patient survival. J Urol 2011;186:1796-1802.

8 Piccirillo JF, Tierney RM, Costas I, Grove L, Spitznagel EL Jr: Prognostic importance of comorbidity in a hospital-based cancer registry. JAMA 2004;291:2441-2447.

$\checkmark 9$ Mayr R, May M, Martini T, et al: Predictive capacity of four comorbidity indices estimating perioperative mortality after radical cystectomy for urothelial carcinoma of the bladder. BJU Int 2012;110:E222-E227.

10 Mayr R, May M, Martini T, et al: Comorbidity and performance indices as predictors of cancer-independent mortality but not of cancer-specific mortality after radical cystectomy for urothelial carcinoma of the bladder. Eur Urol 2012;62:662-670.
11 Sobin LH, Gospodarowicz MK, Wittekind C: International Union against Cancer TNM Classification of Malignant Tumours. Oxford, Wiley-Blackwell, 2010.

12 Bolenz C, Ho R, Nuss GR, et al: Management of elderly patients with urothelial carcinoma of the bladder: guideline concordance and predictors of overall survival. BJU Int 2010; 106:1324-1329.

13 Novotny V, Hakenberg OW, Froehner M, et al: Systematic assessment of complications and outcome of radical cystectomy undertaken with curative intent in patients with comorbidity and over 75 years of age. Urol Int 2013;90:195-201.

14 Harrell FE Jr, Lee KL, Mark DB: Multivariable prognostic models: issues in developing models, evaluating assumptions and adequacy, and measuring and reducing errors. Stat Med 1996;15:361-387.

15 Lachin JM: Power of the Mantel-Haenszel and other tests for discrete or grouped timeto-event data under a chained binomial model. Stat Med 2013;32:220-229. 